\title{
Model-aided optimization and analysis of multi-component catalysts: Application to selective hydrogenation of cinnamaldehyde
}

\author{
Wenjin Yan ${ }^{\mathrm{a}}$, Zhen Guo ${ }^{\mathrm{a}}$, Xinli Jia ${ }^{\mathrm{a}}$, Vinay Kariwala ${ }^{\mathrm{a}}$, Tao Chen ${ }^{\mathrm{b}, *}$, Yanhui Yang, ${ }^{\mathrm{a}, *}$ \\ ${ }^{a}$ School of Chemical and Biomedical Engineering, Nanyang Technological University, Singapore \\ 637459, Singapore \\ ${ }^{b}$ Division of Civil, Chemical and Environmental Engineering, University of Surrey, Guildford GU2 \\ $7 X H, U K$
}

\begin{abstract}
Multi-component catalysts are widely used to exploit the component interactions with the aim to improve catalysis processes. This study applies a model-aided approach to determine the optimal compositions of carbon nanotubes (CNTs) supported $\mathrm{Pt}-\mathrm{Co}-\mathrm{Fe}$ catalysts for selective hydrogenation of cinnamaldehyde. The methodology integrates an iterative response surface methodology (RSM) for optimization, and global sensitivity analysis for interpreting the impact of components and their interactions on the achieved process yield. The RSM encapsulates the state-of-theart space-filling experimental design, advanced data-based modeling, and model-aided optimization while considering prediction uncertainty. A high performance catalyst, $3.4 \% \mathrm{Pt}-1.3 \% \mathrm{Co}-2.6 \% \mathrm{Fe} / \mathrm{CNT}$, is identified with 15 experiments, giving rise to $86.1 \%$ conversion, $86.4 \%$ selectivity and $74.4 \%$ yield. The sensitivity analysis identifies the role of the components and their interactions, which is consistent with reported literature results. For verification purpose, selected catalysts are characterized by using powder X-ray diffraction, transmission electron microscopy, and X-ray photoelectron spectroscopy. Overall, this paper establishes the presented methodology as a powerful tool for design of multi-component catalysts.
\end{abstract}

Keywords:

Catalysis, Catalyst selectivity, Exploration-exploitation, Mathematical modeling, Optimization, Sensitivity analysis

\footnotetext{
*Corresponding author at Division of Civil, Chemical and Environmental Engineering, University of Surrey, Guildford GU2 7XH, UK; Tel.: +44 1483 686593; Fax: +44 1483686581.

** Corresponding author at School of Chemical and Biomedical Engineering, Nanyang Technological University, Singapore 637459, Singapore; Tel.: +65 63168940.

Email addresses: t.chen@surrey.ac.uk (Tao Chen), yhyang@ntu.edu.sg (Yanhui Yang)
} 


\section{Introduction}

Heterogeneous catalysts involving multiple metal (or metal oxide) components have been extensively studied. Compared with single or bimetallic catalysts, multi-component catalysts have demonstrated superior performance in many important chemical reactions, though the catalyst synthesis processes are sometimes more complex. Typical examples include methane oxidative coupling (Huang et al., 2001), preferential CO oxidation (Tompos et al., 2009, 2010), ethanol reforming (Kumar et al., 2011), $\mathrm{NO}_{x}$ conversion (Kaneeda et al., 2010) and cinnamaldehyde (CALD) selective hydrogenation (Guo et al., 2010). The usual approach to development of multi-component catalysts is to adjust the composition of each component in turn while keeping others fixed, in the hope to find the best catalytic performance (typically measured in terms of conversion, selectivity or yield). The resultant catalysts are then subjected to physical/chemical characterization techniques (e.g. X-ray diffraction, transmission electron microscopy and infrared spectroscopy) that are useful to elucidate the reaction mechanisms and to explain the observed performance of catalysts. Nevertheless, this one-factor-at-a-time (OFAT) method has been well recognized to be suboptimal, to say the least, for both catalyst optimization and analysis of results (Myers and Montgomery, 1995; Omata et al., 2006; Serna et al., 2008; Valero et al., 2009; Yan et al., 2011b). The limitation in the data collection process usually does not attain a performance that would otherwise be obtainable by adopting more rational methods. In addition, OFAT method lacks important information regarding how the components interact to provide the observed performance, which might be crucial to guide further investigation. Therefore, a systematic approach using mathematical models is needed to provide guidance for development of catalysts.

In this regard, response surface methodology (RSM), a combination of design of experiments (DoE), empirical modeling and model-based optimization, has become a desired approach to catalyst (and other process/material) development (Omata, 2011; Yan et al., 2011b,a). Compared with the OFAT method, DoE provides more effective coverage of the space of process factors (e.g. the composition of catalyst components and reaction conditions). In early years, DoE was dominated by fractional factorial and central composite designs, assigning two or three levels to each factor (Myers and Montgomery, 1995). The use of limited number of levels per factor was mainly due to the exponential increase of required experiments when the number of factors increases. More recently, it has been shown that better coverage of the factor space can be achieved with a relatively small number of experiments using "space-filling" DoE methods. The idea is to allocate design points (experiments) so that they are distributed within the space as uniformly as possible. Typical methods include Latin hypercube sampling, Hammersley sequence sampling and uniform design; see (Chen and Gao, 2006) for a review. Space-filling designs are also desired for developing flexible data-based models other than the traditional polynomial regression based model (Chen and Gao, 2006).

In addition to appropriate DoE methods, a suitable data-based model is required 
to form the basis for process optimization and analysis. The model approximates the relationship between the factors and response variables (performance measures). The current trend is to replace the traditional polynomial regression with more flexible models that can approximate the actual process more accurately. In the past few years, artificial neural network (ANN) (Huang et al., 2001; Omata et al., 2006; Coleman and Block, 2007), support vector regression (SVR) (Hadjmohammadi and Kamel, 2008) and Gaussian process regression (GPR) (Omata, 2011; Yuan et al., 2008; Yan et al., 2011b,a) have emerged as popular choices. A good model should not only provide accurate prediction, but also faithfully quantify its own prediction uncertainty, which is an important measure to guide process optimization (Jones, 2001). Till date, consensus has not been reached regarding which method is universally better than others, and current empirical studies are restricted to specific cases. Nevertheless, GPR has been shown in a few comparative studies to provide excellent prediction accuracy and reliable quantification of prediction uncertainty (Chen and Gao, 2006; Rasmussen, 1996; Yuan et al., 2008). As a result, GPR is adopted in the present study.

For optimization purpose, a naïve approach would be to search for the catalyst with the best mean performance as predicted by the model. With limited data, the model is normally far from perfect and thus both the prediction mean and its uncertainty should be considered. In experiment-based studies, the role of prediction uncertainty is unfortunately often under-emphasized or even simply ignored. Conceptually, a large uncertainty indicates lack of exploration of that region, and more data should be collected to improve the model. This represents the classical example of model exploitation (where prediction mean is used to find the best performance) and factor space exploration (where experiments are allocated to uncertain regions). In the literature, model exploitation and space exploration have been successfully combined by using the statistical measure of expected improvement (EI) (Jones, 2001). EI has been applied for model-aided optimization of various catalytic processes, including epoxidation of trans-stilbene (Yan et al., 2011a), CO oxidation (Yan et al., 2011b), and the Friedel-Crafts reaction (Omata, 2011). In this work, EI is implemented for the purpose of reliable optimization.

Following optimization, the analysis and interpretation of the results, in terms of how the factors affect the catalyst performance, are equally important. With limited data, model-aided local sensitivity analysis (SA) has been widely used for this purpose (Tang et al., 2010; Yuan et al., 2008). Mathematically, SA studies how the variation in the factors $\left(\mathbf{x}=\left[x_{1}, \ldots, x_{d}\right]\right.$ where $d$ is the number of factors) contributes to the variation in the response $(y)$ (Saltelli et al., 2008). Local SA relies on the partial derivatives $\partial y / \partial x_{i}, i=1, \ldots, d$, which are evaluated at a certain nominal point $\mathbf{x}_{0}$ (e.g. the best component compositions of a catalyst). Local SA is essentially an OFAT method, and is known to be ineffective for non-linear processes (Saltelli et al., 2008). In contrast, global SA evaluates the factors' effect within the entire design space, giving rise to a global picture of the process being investigated. Global SA has seen increasing applications in chemical engineering, in particular for the analysis of first-principles models (McRae et al., 1982; Haaker and Verheijen, 2004; Chhatre et al., 
2008; Degerman et al., 2009), and to a lesser extent for data-based models (Chen and Yang, 2011).

In the present work, we demonstrate the application of modeling tools for the development and analysis of multi-component catalysts. A previously studied reaction, selective hydrogenation of cinnamaldehyde (CALD) to cinnamal alcohol (CALC) (Guo et al., 2010), is adopted as case study for its important role in the pharmaceutical and fragrance industries (Gallezot and Richard, 1998). The catalyst support has been chosen to be carbon nanotubes (CNTs) due to their proven superior performance. For the active phase, Pt serves as reaction sites while Co and Fe are promoters. The modeling method has previously been applied to optimize reaction conditions like temperature and reaction time (Yan et al., 2011b). The main challenge here is that for a certain catalyst, testing reaction conditions is usually fast (several hours per experiment), whereas synthesizing catalysts is slow (around several days per catalyst). Therefore, the number of experiments that are practically affordable is limited (to no more than 20 in our laboratory). One aim of this study is thus to verify the applicability of the modeling approach to the optimization of composition of multi-component catalysts whereby very limited data can be collected. More important contribution of this paper is the introduction of global SA to analyze the impact of multiple components on the obtained performance, including both factors' main effects and the interactions between factors. It should be noted that the term "interaction" is used in this paper in a statistical sense and refers to the fact that the response variable is not affected independently by individual factors, but relies on the combined effect of factors (Saltelli et al., 2008). Statistical interaction is not equivalent to the physical interaction where multiple components form new alloys with modified catalytic activity. Nevertheless, statistical interaction is a useful indication of physical interaction, as the latter can only be verified by expensive and time-consuming characterization techniques. In this sense, the proposed methodology is complementary to physical catalyst characterization to obtain useful information about the process. In the rest of this paper, the term "interaction" is used in a statistical sense unless otherwise stated.

It is worth pointing out that apart from data-based methods, the reaction system, selective hydrogenation of CALD, has been studied using mechanistic (kinetic) models (Toebes et al., 2005; Vergunst et al., 2001), which can then be employed to aid catalyst optimization. The underlying physical/chemical basis of mechanistic models is clearly desired, and it usually results in better extrapolation capability. Nevertheless, databased empirical models are still widely used especially by practitioners: they are costefficient to develop and may be more suitable for exploring a range of different catalysts. This paper aims to provide a better solution for empirical model-based catalyst design, and thus rigorous comparison between empirical and mechanistic models is not within the scope.

The rest of this paper is organized as follows. Section 2 outlines the overall modeling method and its application in catalyst optimization. The experimental details of selective hydrogenation of CALD to CALC, and catalyst characterization, are introduced in Section 3. Section 4 presents the results of model-aided catalyst optimization 
and analysis. Finally, Section 5 concludes this paper.

\section{Methods}

The overall framework of the model-aided optimization and analysis approach includes:

1. DoE to allocate appropriate initial experiments.

2. Development of a data-based model from all available experimental data.

3. Model-based optimization to allocate experimental point(s) for the next iteration, and collect additional data from experiment(s).

4. Global SA of the final model for analysis.

Steps 1-3 form the RSM, which iterates between Steps 2 and 3 until a satisfactory catalyst is found or experimental resources run out. Finally, global SA is utilized for process analysis. These four steps are presented in detail subsequently.

\subsection{Design of Experiments}

DoE is becoming a standard element to aid the development of catalysts. The main objective is to provide good coverage of the factor space with a limited number of experiments. The distribution of data has a crucial impact on the quality of the model. Nevertheless, the majority of reported studies in the literature still rely on the traditional fractional factorial and central composite designs (or their variants), which are purposely designed for polynomial regression (Myers and Montgomery, 1995) but less suitable for more complex models. In contrast, the concept of space-filling was proposed to cover the factor space as uniformly as possible within each dimension. Due to the limited experiments that can be allocated, straightforward random sampling is not useful since it requires a large number of samples. Instead, stratified and deterministic sampling methods have been used for this purpose, such as Latin hypercube sampling (LHS) (McKay et al., 2000), uniform design (Fang et al., 2000) and Hammersley sequence sampling (HSS) (Kalagnanam and Diwekar, 1997). Among these methods, HSS attains excellent uniformity while retaining simplicity in implementation (Chen and Gao, 2006), and is adopted in this work. The Hammersley sequence falls within the family of number-theoretic approaches for constructing the low-discrepancy sequences that occupy the factor space uniformly. The detailed definition and implementation of HSS is available in (Kalagnanam and Diwekar, 1997).

\subsection{Modeling using GPR}

The model plays a nexus role in RSM; it forms the basis for systematic (as opposed to trial-and-error) optimization and analysis. The choice of GPR for modeling is not only due to its outstanding accuracy, but also due to the automatic provision of prediction variance as a measure of uncertainty (Rasmussen, 1996; Chen and Gao, 2006; Yuan et al., 2008). Formally, for a set of experimental data with size $n:\left\{\mathbf{x}_{i}, y_{i} ; i=1, \ldots, n\right\}$, 
a GPR is defined for the regression function $y(\mathbf{x})$ with a zero-mean Gaussian distribution $\mathbf{y}=\left(y_{1}, \ldots, y_{n}\right)^{\mathrm{T}} \sim G(\mathbf{0}, \mathbf{C})$, where $\mathbf{C}$ is the $n \times n$ covariance matrix. The $i j$-th element of the covariance matrix is further parameterized by a covariance function $\mathbf{C}_{i j}=C\left(\mathbf{x}_{i}, \mathbf{x}_{j}\right)$. A commonly used form of covariance function is implemented in this study (Rasmussen and Williams, 2006):

$$
C\left(\mathbf{x}_{i}, \mathbf{x}_{j}\right)=a_{0}+a_{1} \sum_{k=1}^{d} x_{i k} x_{j k}+v_{0} \exp \left(-\sum_{k=1}^{d} w_{k}\left(x_{i k}-x_{j k}\right)^{2}\right)+\sigma^{2} \delta_{i j}
$$

where $x_{i k}$ is the $k$-th variable (factor) of $\mathbf{x}_{i} ; \delta(\cdot)$ is the delta function: $\delta_{i j}=1$ if $i=j$, otherwise $\delta_{i j}=0$. The four terms in Eq. (1) reflect the effect of constant bias, linear correlation, non-linear correlation and random noise, respectively, enclosing the most common forms of covariance that are encountered in practice. It was shown to give excellent prediction when compared with other forms (Rasmussen, 1996). The parameters, $\boldsymbol{\theta}=\left(a_{0}, a_{1}, v_{0}, w_{1}, \ldots, w_{d}, \sigma^{2}\right)^{\mathrm{T}}$, can be estimated by maximizing the logarithm of the likelihood function using, for example, the conjugate gradient optimization method. In this study, a Matlab implementation of GPR is used, which is publicly available from http://www.gaussianprocess.org/gpml/code/matlab/doc/ (Rasmussen and Williams, 2006).

With the estimated parameters, the prediction for a new data point $\mathbf{x}^{*}$ is also Gaussian distributed with the following mean $\left(\hat{y}^{*}\right)$ and variance $\left(\sigma_{\hat{y}^{*}}^{2}\right)$ :

$$
\begin{aligned}
\hat{y}^{*} & =\mathbf{k}^{\mathrm{T}}\left(\mathbf{x}^{*}\right) \mathbf{C}^{-1} \mathbf{y} \\
\sigma_{\hat{y}^{*}}^{2} & =C\left(\mathbf{x}^{*}, \mathbf{x}^{*}\right)-\mathbf{k}^{\mathrm{T}}\left(\mathbf{x}^{*}\right) \mathbf{C}^{-1} \mathbf{k}\left(\mathbf{x}^{*}\right)
\end{aligned}
$$

where $\mathbf{k}\left(\mathbf{x}^{*}\right)=\left[C\left(\mathbf{x}^{*}, \mathbf{x}_{1}\right), \ldots, C\left(\mathbf{x}^{*}, \mathbf{x}_{n}\right)\right]^{\mathrm{T}}$.

\subsection{Optimization}

For a chemical process, the usual objective is to maximize a certain response variable (e.g. conversion, selectivity or yield). Given a model, a straightforward method is to experiment at $\mathrm{x}^{*}$ that has the largest predicted mean. With the additional experimental data, the model is then updated by including all available data, prior to being used for making new predictions. This iterative procedure terminates when process improvement decreases below a threshold. However, this approach ignores the uncertainty in model prediction, and thus the identified "optimum" is likely to be a local one (Jones, 2001). To obtain better performance, the prediction mean and variance should be jointly considered, since the variance indicates the lack of exploration of the region and the need for more experiments. In the literature, several methods have been investigated to handle prediction variance, including maximization of lower or upper prediction bound (Yuan et al., 2008; Tang et al., 2010), minimization of information free energy (Lin and Jang, 1998), maximization of relative information gain (Coleman and Block, 2007), and maximization of expected improvement (EI) (Jones, 2001). Among these methods, EI is based on a rigorous statistical formulation and does not require 
user-determined weights as other methods do. Therefore, the EI method is adopted in this work to allocate the next experiment $\mathrm{x}^{*}$ with the aim of optimizing the process.

To describe the EI method, suppose that $y(\mathbf{x})$ is the prediction at $\mathbf{x}$ from the current GPR model and the best experimental result obtained so far is $f_{\text {best }}$. Thus, the improvement at $\mathbf{x}$ is $I(\mathbf{x})=y(\mathbf{x})-f_{\text {best. }}$. According to Eqs. (2)-(3), $y(\mathbf{x})$ is Gaussian distributed with mean $\hat{y}$ and variance $\sigma_{\hat{y}}^{2}$. Therefore, the improvement $I(\mathbf{x})$ is also Gaussian distributed with mean $\hat{y}-f_{\text {best }}$ and the same variance as $\hat{y}$, and the expectation of $I$ is given by $\int_{-\infty}^{\infty} I p(I) d I$, where $p(\cdot)$ is the probability density function. However, improvement over the best result, $f_{\text {best }}$, requires a positive $I$, and thus the integration should start from 0. Accordingly, EI is defined as

$$
\mathrm{EI}(\mathbf{x})=E[\max \{0, I(\mathbf{x})\}]=\int_{0}^{\infty} I p(I) \mathrm{d} I
$$

where $E(\cdot)$ is the expectation operator. Substituting the probability density function of Gaussian distribution and applying integration by parts, EI can be analytically calculated as (Jones, 2001):

$$
\mathrm{EI}(\mathbf{x})=\sigma_{\hat{y}}[u \Phi(u)+\phi(u)]
$$

where $u=\left(\hat{y}-f_{\text {best }}\right) / \sigma_{\hat{y}} ; \Phi(\cdot)$ and $\phi(\cdot)$ denote the cumulative distribution function and density function of the standard normal distribution, respectively. As a result, EI is large when the predictive mean is higher than $f_{\text {best }}$ and/or the prediction variance is large. For the purpose of iterative optimization, the next experiment is conducted at $\mathbf{x}$ that maximizes the EI, and we denote $\mathrm{EI}_{\max }=\max _{\mathbf{x}} \mathrm{EI}(\mathbf{x})$.

\subsection{Global sensitivity analysis}

Model-based global SA is to decompose the model $y(\mathbf{x})$ into main effects and interactions (Saltelli et al., 2008). The main effects correspond to the impact of individual factors $x_{i}(i=1, \ldots, d)$, while the interactions quantify the joint impact of multiple factors. The decomposition is given as

$$
\begin{aligned}
y(\mathbf{x})= & E(y)+\sum_{i=1}^{d} z_{i}\left(x_{i}\right)+\sum_{1 \leq i<j \leq d} z_{i, j}\left(\mathbf{x}_{i, j}\right)+\sum_{1 \leq i<j<k \leq d} z_{i, j, k}\left(\mathbf{x}_{i, j, k}\right) \\
& +\ldots+z_{1,2, \ldots, d}(\mathbf{x})
\end{aligned}
$$

where $E(\cdot)$ denotes the expectation and $E(y)$ is the overall mean of the model; $\mathbf{x}_{i, j}=$ $\left(x_{i}, x_{j}\right)^{\mathrm{T}}$ and $\mathbf{x}_{i, j, k}=\left(x_{i}, x_{j}, x_{k}\right)^{\mathrm{T}}$. In addition, the main effects are

$$
z_{i}\left(x_{i}\right)=E\left(y \mid x_{i}\right)-E(y)
$$

and the second order interactions are

$$
z_{i, j}\left(\mathbf{x}_{i, j}\right)=E\left(y \mid \mathbf{x}_{i, j}\right)-z_{i}\left(x_{i}\right)-z_{j}\left(x_{j}\right)-E(y)
$$


The expression for high-order interactions can be derived similarly. Furthermore, to quantify the importance of factors and their interactions, a variance-based measure is utilized:

$$
\begin{aligned}
V_{i} & =\operatorname{var}\left\{z_{i}\left(x_{i}\right)\right\}=\operatorname{var}\left\{E\left(y \mid x_{i}\right)\right\} \\
V_{i, j} & =\operatorname{var}\left\{z_{i, j}\left(\mathbf{x}_{i, j}\right)\right\}=\operatorname{var}\left\{E\left(y \mid \mathbf{x}_{i, j}\right)\right\}-V_{i}-V_{j}
\end{aligned}
$$

By dividing the total variance of $y$, the $V_{i}$ and $V_{i, j}$ are normalized to the range $[0,1]$, giving rise to the sensitivity indices (SI): $S_{i}=V_{i} / V, S_{i, j}=V_{i, j} / V$, and so on. Note that the sum of all SIs is unity.

Another useful measure is the variance of total effect for the $i$-th factor:

$$
V_{T_{i}}=V-\operatorname{var}\left\{E\left(y \mid \mathbf{x}_{-i}\right)\right\}
$$

where $\mathbf{x}_{-i}$ is the sub-vector of $\mathbf{x}$ obtained after removing $x_{i}$. The corresponding SI is $S_{T_{i}}=V_{T_{i}} / V$. The total effect index accounts for the total contribution to the response variable due to factor $x_{i}$, including its main effect plus all higher-order interactions. Therefore, $S_{T_{i}} \geq S_{i}$, where equality indicates the absence of interaction with other factors.

The computation of the SIs mainly involves the evaluation of the integrals when calculating the expectations and variances in Eqs. (7)-(10). An efficient Monte Carlo method, originally proposed by Sobol', has been widely used for this purpose; see (Sobol, 2001; Saltelli et al., 2008) for the detailed algorithm. Besides the quantitative SIs, a plot of the main effects $E\left(y \mid x_{i}\right)$ and the interactions $E\left(y \mid x_{i}, x_{j}\right)$ versus the process factors is useful to graphically illustrate the impact of process factors. These effects can be calculated based on the Monte Carlo method described in (Saltelli et al., 2008; Chen and Yang, 2011). An in-house Matlab program was implemented to produce the results reported in this paper.

\section{Experimental}

The proposed model-aided optimization and analysis methodology is applied to the development of multi-component catalysts for selective hydrogenation of CALD to CALC. The catalyst under consideration is Pt-Co-Fe/CNT where the composition of the three metal components needs to be optimized. CALD is an $\alpha, \beta$-unsaturated aldehyde. Three main products can be obtained by hydrogenating $\mathrm{C}=\mathrm{O}$ bond and/or $\mathrm{C}=\mathrm{C}$ bond: cinnamal alcohol (CALC), hydrocinnamylalcohol (HALC) and hydrocinnamaldehyde (HALD). Small amount of other by-products, like acetal, may also be produced. The schematic of the reaction pathways is shown in Figure 1. As CALC is the desired product, the conversion, selectivity and yield of this reaction are calculated as 


$$
\begin{aligned}
\text { Conversion } & =\frac{\text { moles of reactant converted }}{\text { moles of reactant in feed }} \\
\text { Selectivity } & =\frac{\text { moles of CALC formed }}{\text { moles of reactant converted }} \\
\text { Yield } & =\text { Conversion } \times \text { Selectivity }
\end{aligned}
$$

where the yield is chosen as the ultimate response variable to be maximized in this study. The rest of this section presents the experimental details.

(Figure 1 about here)

\subsection{Catalyst synthesis}

The chemicals involved in catalyst synthesis include $\mathrm{H}_{2} \mathrm{PtCl}_{6} \cdot 6 \mathrm{H}_{2} \mathrm{O}(>37.5 \% \mathrm{Pt}$ basis, Sigma-Aldrich), $\mathrm{Co}\left(\mathrm{NO}_{3}\right)_{2} \cdot 6 \mathrm{H}_{2} \mathrm{O}$ (>98\%, Sigma-Aldrich), $\mathrm{Fe}\left(\mathrm{NO}_{3}\right)_{3} \cdot 9 \mathrm{H}_{2} \mathrm{O}(>98 \%$, Sigma-Aldrich), ethylene glycol (EG, 99.5\%, Sinopharm Chemical Reagent), tetradecane $(>99.5 \%$, Fluka), and ethyl acetate (EA, $>99.5 \%$, Acros). These chemicals were used as received without any further purification. Pristine CNT (>95\%, Cnano) was treated with nitric acid according to the procedures described in (Lordi et al., 2001). This pretreatment removes amorphous carbonaceous and metallic impurities, and facilitates the creation of abundant surface functional groups to help uniform deposition of metal precursors. First, $100 \mathrm{mg}$ of functionalized CNT was weighed and aqueous solution of $\mathrm{H}_{2} \mathrm{PtCl}_{6} \cdot 6 \mathrm{H}_{2} \mathrm{O}, \mathrm{Co}\left(\mathrm{NO}_{3}\right)_{2} \cdot 6 \mathrm{H}_{2} \mathrm{O}$ and $\mathrm{Fe}\left(\mathrm{NO}_{3}\right)_{3} \cdot 9 \mathrm{H}_{2} \mathrm{O}$ were added. After short sonication, the mixture was dried at $373 \mathrm{~K}$ overnight. Then, $40 \mathrm{~mL}$ of EG was added to the composite, followed by $10 \mathrm{~min}$ of sonication to facilitate uniform suspension. The suspension was transferred into a three-neck flask with condenser and placed into a microwave reactor (MAS-II, Sineo). With magnetic stirrer and infrared temperature monitor, the suspension was heated to $438 \mathrm{~K}$ in 0.5 min and kept at that temperature for $1.5 \mathrm{~min}$. After cooling for $30 \mathrm{~min}$, the powder was filtered and washed with 150 $\mathrm{mL}$ deionized water three times. The final catalyst was obtained after the residue was dried at $333 \mathrm{~K}$ overnight.

\subsection{Selective hydrogenation of $C A L D$}

The reaction was carried out in a stainless steel reactor with Teflon liner (Parr 4950, controller 4843). First, $10 \mathrm{mg}$ of catalyst, $200 \mathrm{mg}$ of CALD (1.5 mmol, >99\%, Aldrich), $7 \mathrm{~mL}$ of EA and $38 \mu \mathrm{L}$ of tetradecane $(0.146 \mathrm{mmol})$ were loaded into the liner, where EA acted as solvent and tetradecane as an internal standard. Then, the air residue in the liner was expelled by pressurizing and releasing by 10 atm $\mathrm{H}_{2}$ three times. The reaction was carried out at $333 \mathrm{~K}$ under 10 atm $\mathrm{H}_{2}$ for $1 \mathrm{~h}$ with stirring speed $600 \mathrm{rpm}$. After the reaction, catalyst powder was filtered off while the filtrate was retained for gas chromatograph analysis. An online GC-6890N (Agilent Technologies) equipped with an HP-5 column (Agilent) was used for the analysis, and the signal of flame ionization detector was used for conversion and selectivity calculation. Repeated experiments during preliminary investigation showed that the $95 \%$ confidence bound for the obtained yield is approximately $\pm 0.5 \%$. 


\subsection{Catalyst characterization}

Powder X-ray diffraction (XRD) patterns were recorded on a Bruker D8 advance powder diffractometer at ambient conditions, using filtered $\mathrm{Cu}-\mathrm{K} \alpha$ radiation source $(\lambda$ $=1.54056 \AA$ A $)$ operated at $40 \mathrm{kV}$ and $40 \mathrm{~mA}$. Diffraction data were collected from 10 to $80^{\circ}(2 \theta)$ at a scanning speed of $2^{\circ} / \mathrm{min}$. Prior to the test, samples were dried at $100{ }^{\circ} \mathrm{C}$ overnight.

Transmission electron microscopy (TEM) measurements were performed on a JEOL JEM-1400 system operated at $100 \mathrm{kV}$. The samples were suspended in ethanol and dispersed on a holey carbon-coated $\mathrm{Cu}$ grid. The mean particle diameter was calculated from the mean frequency distribution by counting approximately 200 particles.

X-ray photoelectron spectroscopy (XPS) was performed on a VG Escalab 250 spectrometer equipped with an $\mathrm{Al}$ anode $(\mathrm{Al} \mathrm{K} \alpha=1486.6 \mathrm{eV})$. The background pressure in the analysis chamber was lower than $1 \times 10^{-7} \mathrm{~Pa}$. Measurements were taken using $20 \mathrm{eV}$ pass energy, $0.1 \mathrm{eV}$ step and $0.15 \mathrm{~min}$ dwelling time. The correction of the binding energy $(\mathrm{BE})$ employed the $\mathrm{C}_{1 \mathrm{~s}}$ peak of adventitious $\mathrm{C}$ at $284.6 \mathrm{eV}$. The background contribution caused by inelastic process was obtained by the Shirley method and subtracted.

\section{Results}

\subsection{Yield optimization}

The initial DoE, using HSS, was used to allocate 10 experiments. Three factors were considered, namely the percentage loading in mass of $\mathrm{Pt}, \mathrm{Co}$ and $\mathrm{Fe}$, with the corresponding range being 3-5\%,1-3\% and 1-3\%, respectively. The range was decided from our prior experience in this system. Excessive Pt is known to have adverse impact on selectivity. In addition, it appeared that when the overall loading of promoter (both $\mathrm{Co}$ and $\mathrm{Fe}$ ) was more than twice of that of $\mathrm{Pt}$, the reactivity dramatically reduced because the promoter covered active sites. Thus the lower limit of Pt was determined to be the upper limit of each promoter $(3 \%)$. The range defined the factor space to be explored, and was determined to be reasonable by referring to similar studies in the literature (Guo et al., 2010). The number of initial experiments should be determined by considering the number of factors, range of each factor, the complexity of the response surface, and the cost of experiment. Of these, the complexity of the response surface is not known prior to actual experiments. Clearly, more experiments are desired to improve model accuracy. The choice of 10 is comparable to those from conventional DoE methods; for example the two-level factorial design for three factors gives $2^{3}=8$ experiments. This choice is also due to the resource constraint in our laboratory. The results of initial experiments are given in Table 1 . The best catalyst found so far was $3.4 \% \mathrm{Pt}-2.9 \% \mathrm{Co}-1.2 \% \mathrm{Fe} / \mathrm{CNT}$ (No. 8), with a yield of 0.647 .

(Table 1 about here)

After obtaining the initial data, the proposed iterative modeling and optimization procedure was followed to search for the next experiment that had the potential to 
further improve the yield. The results of the six subsequent iterations are listed in Table 2. Initially, a GPR model was developed from the 10 data points to model the relationship between the factors and the yield. The calculated maximal EI (the column "EI $\mathrm{max}_{\text {" }}$ in the table) was 0.223 , indicating a relatively large potential for further improvement. The composition of the design point corresponding to the maximal EI is given in the columns of " $x_{1}$ ", " $x_{2}$ " and " $x_{3}$ " in Table 2 , for which the $95 \%$ confidence bound of predicted yield was $\hat{y}=0.569 \pm 0.653$. Considering the best yield found so far (0.647), the mean prediction of this design point did not seem promising; it was selected mainly to explore the factor space because of the large prediction uncertainty. The actual experimental result, given in the last three columns in the table, indicated that indeed the design No. 11 was inferior to what had been achieved. However, this experiment should not be viewed as a waste of resource; rather it allowed gathering more information about the process to improve the model.

\section{(Table 2 about here)}

Subsequently, a GPR model was re-built with 11 data points, and the iterative procedure was repeated until experiment No. 15. In retrospect, it appeared that all experiments except No. 15 were designed mainly for exploration, since the mean predictions were always below the best result seen up to that iteration. In addition, the exploration at experiment No. 11-13 did not result in actual improvement, while experiment No. 14 did. The process yield was further improved to 0.744 at experiment No. 15, and the prediction mean was the same as the actual yield, which was believed to be a coincidence. Furthermore, the maximal EI decreased throughout the iterative procedure, indicating gradually reduced likelihood of process improvement. The designed experiment No. 16 had a maximal EI of only 0.011 , which in conjunction with the prediction performance of experiment No. 15, led to the decision to terminate at this iteration. The best catalyst obtained through this procedure was $3.4 \% \mathrm{Pt}-1.3 \% \mathrm{Co}-$ $2.6 \% \mathrm{Fe} / \mathrm{CNT}$, which exhibited desirable conversion (0.861), selectivity (0.864), and yield (0.744).

It is difficult to determine whether the designed catalyst is globally optimal for the Pt-Co-Fe/CNT system. In theory, if an infinite number of iterations are allowed, the procedure guarantees to find the global optimum of the process (Jones, 2001). Nevertheless, this theoretical result cannot be verified in practical process development.

\subsection{Sensitivity analysis}

The GPR models developed based on the entire 15 experiments were used to elucidate the impact of component composition on catalyst performance. Three models were developed separately for different response variables, namely conversion, selectivity and yield. For each GPR model, global SA was implemented to calculate the SIs. It was found that 10,000 Monte Carlo samples were sufficient for accurate approximation of the integrals while being computationally manageable (Chen and Yang, 2011). The main effects due to the three components are shown in Table 3 (both $S_{i}$ and $S_{T_{i}}$ ). 
For conversion, Pt clearly gained high indices, confirming its pivotal role for CALD hydrogenation (Lordi et al., 2001). The SIs of Fe showed its intermediate impact on conversion, while Co's effect appeared to be negligible. In contrast, the main effects on selectivity were quite different from those on conversion. Fe played a leading role, which may be explained by its moderate hydrogenation capability (Ishihara, 1987; Tihay et al., 2002). Pt showed a relatively weak effect on selectivity as its hydrogenation ability may be excessively strong (Gallezot and Richard, 1998; Liu et al., 2008). Finally, Co had nearly no influence on reaction selectivity. The yield, being a product of conversion and selectivity, was strongly affected by $\mathrm{Pt}$ and $\mathrm{Fe}$, but not Co per se. However, the total SI $\left(S_{T_{i}}\right)$ of Co for yield is close to 0.1, indicating its non-negligible, albeit small, impact due to interactions with other factors.

\section{(Table 3 about here)}

SIs quantify the magnitude of the factors' influence, but not the trend. A graphical illustration of the main effects versus various factors, as shown in Figure 2, is a useful complement to SIs. For conversion, the graph reinforced the observation that Co and Fe loadings had weak impact, while Fe gave a slightly positive impact especially at high loadings. Note the factors are scaled to lie in the range $[0,1]$ in Figure 2. Pt surprisingly showed a sharp drop of conversion rate at an intermediate level (around 3.8 wt. \%), which needs further investigation to explain. For selectivity, Pt loading showed a negative impact, as its hydrogenation capacity is too strong to obtain CALC. Co loading still played an unimportant role in terms of main effect, while the impact of Fe loading was clearly positive on selectivity. The graph for yield illustrates the combined effects of conversion and selectivity, where Co loading had negligible main effect which is consistent with the SIs.

(Figure 2 about here)

Different from $S_{i}, S_{T_{i}}$ includes not only the main effect but also the effects from related interactions. From the differences between $S_{i}$ and $S_{T_{i}}$ shown in Table 3, we can conclude that there are important effects of Pt-related interactions and Co-related interactions on conversion, while there are also important effects of Pt-related interactions and Fe-related interactions on selectivity. This observation is validated by second-order interactions subsequently.

In the development of multi-component catalysts, the interactions between components and their impact on catalyst performance is an interesting and important topic, since the main effects are generally similar to the performance of corresponding singlecomponent catalysts. Global SA is a useful tool for this purpose. The SI of second order interactions, $S_{\mathrm{PtCo}}, S_{\mathrm{PtFe}}$ and $S_{\mathrm{CoFe}}$, are listed in Table 4 . The Pt-Co interaction in Pt-Co-Fe/CNT catalysts showed significant impact on CALD conversion, a phenomenon that was reported in Pt-Co bimetallic catalysts (Lu et al., 1992; Schanke et al., 1995). The Pt-Fe interaction improves reaction selectivity significantly, similar to the reported studies on Pt-Fe catalysts (Beccat et al., 1990; Fukuoka et al., 1990; 
Crabb and Marshall, 2001). As a consequence, these two forms of interactions showed significant impact on process yield. Contrary to reportedly good performance of $\mathrm{Co}-\mathrm{Fe}$ catalysts (Ishihara, 1987; Tihay et al., 2002), the Co-Fe interaction in Pt-Co-Fe/CNT catalysts was insignificant, probably due to the fact that its effect was overshadowed by the presence of Pt. This observation also illustrates that model-aided global SA is useful to analyze catalysts with more than two components, which might be too complex for purely experiment-based analysis. In addition, the SIs for third-order interaction, $S_{\mathrm{PtCoFe}}$, were $0.011,0.020$ and 0.023 for conversion, selectivity and yield, respectively, indicating insignificant impact.

(Table 4 about here)

Figures 3, 4 and 5 demonstrate the expected response surface as a function of factor pairs, $E\left(y \mid x_{i}, x_{j}\right)$, which encapsulates both main effects and second-order interactions. These graphs largely confirm/reinforce the results of analysis done so far. Comparing the effects of Pt-Co and Pt-Fe on conversion (Figure 3), it can be observed that the response does vary to some extent with the Co loading, but remains almost constant with variation in Fe loading. In addition, the Co-Fe graph is almost flat, indicating their extremely weak impact on conversion when compared with Pt. Similarly, the dominance of Fe loading on selectivity can be seen in Figure 4. However, the response surface for Pt-Fe does not completely depends on Fe loading, but also slightly correlates with Pt loading, indicating some interactions between these two components. In contrast, the selectivity versus Fe almost follows the same trend regardless of the value of Co loading (Figure 4, response surface for Co-Fe), suggesting negligible interaction between Co and Fe loadings. Finally, yield is primarily affected by the main effects Pt and Fe, and to a significantly less extent by the interactions Pt-Fe and Pt-Co, but not Co alone, as can be inferred from both Figure 5 and the SIs in Tables 3 and 4.

(Figures 3, 4 and 5 about here)

\subsection{Characterization}

Four representative catalysts in Table 2 were characterized, including the optimal one (No. 15), and No. 11-13 for comparison purpose. Inductive coupled plasma (ICP) was employed to confirm the metal loadings.

XRD patterns are included in Figure 6 . The strong peak at $2 \theta=25.8^{\circ}$ corresponds to $\left(\begin{array}{lll}0 & 0 & 2\end{array}\right)$ diffraction of hexagonal graphite (Guo et al., 2010), suggesting that graphite structure of CNT is preserved after series of pretreatments. The peaks at $2 \theta=39.7^{\circ}$ and $2 \theta=46.2^{\circ}$ were related to cubic platinum structure (Chytil et al., 2009). The average diameter of $\mathrm{Pt}$ particles was calculated using the Scherrer equation and is shown in Table 5, based on full width of half the maximum intensity of Pt $\left(\begin{array}{lll}1 & 1 & 1\end{array}\right)$ reflection.

(Figure 6 and Table 5 about here) 
TEM images directly show the size and size distribution of Pt nanoparticles, as given in Figure 7. The average diameter calculated from TEM is similar to that from XRD. No significant variation of particle size is observed over the four samples. Nonetheless, decreasing Pt loading and increasing Fe/Co loading result in smaller particle sizes, implying that metal oxides such as $\mathrm{FeO}_{\mathrm{x}}$ and $\mathrm{CoO}_{\mathrm{x}}$ may enhance the dispersion of $\mathrm{Pt}$ metal nanoparticles, and thus the reaction yield.

(Figure 7 about here)

XPS spectra were measured to investigate the oxidation state of $\mathrm{Pt}$, Co and Fe. In Figure 8, Co and Fe elements in these catalysts are nearly all in oxide form. $\mathrm{Pt}$ are generally in metallic state, and its binding energy varies with different loading of promoter, suggesting strong interaction between $\mathrm{Pt}$ active sites and $\mathrm{Fe} / \mathrm{Co}$ promoters.

(Figure 8 about here)

\section{Conclusions}

This work presented a model-aided catalyst development and analysis methodology, with application to search for the optimal compositions of a multi-component catalyst (Pt-Co-Fe/CNT) for selective cinnamaldehyde hydrogenation. Initially, ten experiments were allocated by using Hammersley sequence sampling, a space-filling experimental design method, to provide reasonable coverage of the factor space. After five iterations of modeling and model-aided optimization, the $3.4 \% \mathrm{Pt}-1.3 \% \mathrm{Co}-2.6 \% \mathrm{Fe} / \mathrm{CNT}$ catalyst was obtained, with 0.861 conversion, 0.864 selectivity and 0.744 yield, which was a significant improvement over the best yield (0.647) obtained during initial experiments. Afterwards, global sensitivity analysis was adopted to evaluate the effect of individual factors (the loadings of the three components) and their interactions on catalyst performance, a task that would be otherwise impractical if the one-factor-ata-time approach is used. The resulting observations are qualitatively consistent with reported studies in the literature, including (a) Pt is crucial for conversion, (b) Fe is important for selectivity, and (c) the interactions Pt-Co and Pt-Fe further promote conversion and selectivity, respectively.

For verification purpose, selected catalysts were characterized by XRD, TEM and XPS, which further confirmed the observed interactions. The analysis also highlighted a phenomenon, i.e. the sharp drop of conversion when $\mathrm{Pt}$ is around 4 wt. \%, which cannot be explained solely based on the GPR model and requires further investigation.

In the present study, global SA was conducted after catalyst optimization stage. It is possible to carry out SA during each iteration of the optimization stage, in order to provide further guidance to optimization. This approach will be investigated. In conclusion, we envisage that the integrated optimization and analysis methodology will be useful if catalysts with even more components are to be developed. 


\section{Acknowledgment}

This work was partially supported by the Singapore AcRF Tier 1 Grant (RG 19/09) and the A*STAR SERC Grant (102 101 0020).

\section{References}

Beccat, P., Bertolini, J. C., Gauthier, Y., Massardier, J., Ruiz, P., 1990. Crotonaldehyde and methylcrotonaldehyde hydrogenation over Pt(111) and Pt80Fe20(111) single crystals. Journal of Catalysis 126, 451-456.

Chen, T., Yang, Y. H., 2011. Interpretation of non-linear empirical data-based process models using global sensitivity analysis. Chemometrics and Intelligent Laboratory Systems 107 (1), 116-123.

Chen, X., Gao, F. R., 2006. Profiling of injection velocity for uniform mold filling. Advances in Polymer Technology 25 (1), 13-21.

Chhatre, S., Francis, R., Newcombe, A., Zhou, Y., Titchener-Hooker, N., King, J., Keshavarz-Moore, E., 2008. Global sensitivity analysis for the determination of parameter importance in the chromatographic purification of polyclonal antibodies. Journal of Chemical Technology and Biotechnology 83, 201-208.

Chytil, S., Glomm, W. R., Blekkan, E. A., 2009. Characterization of Pt/SBA-15 prepared by the deposition-precipitation method. Catalysis Today 147 (3-4), 217-223.

Coleman, M. C., Block, D. E., 2007. Nonlinear experimental design using Bayesian regularized neural networks. AIChE Journal 53 (6), 1496-1509.

Crabb, E. M., Marshall, R., 2001. Properties of alumina supported Pd-Fe and Pt-Fe catalysts prepared using surface organometallic chemistry. Applied Catalysis A: General 217 (1-2), 41-53.

Degerman, M., Westerberg, K., Nilsson, B., 2009. A Model-Based Approach to Determine the Design Space of Preparative Chromatography. Chemical Engineering and Technology 32, 1195-1202.

Fang, K. T., Lin, D. K. J., Winker, P., Zhang, Y., 2000. Uniform design: theory and application. Technometrics 42, 237-248.

Fukuoka, A., Kimura, T., Kosugi, N., Kuroda, H., Minai, Y., Sakai, Y., Tominaga, T., Ichikawa, M., 1990. Bimetallic promotion of alcohol production in CO hydrogenation and olefin hydroformylation on RhFe, PtFe, PdFe, and IrFe cluster-derived catalysts. Journal of Catalysis 126 (2), 434-450.

Gallezot, P., Richard, D., 1998. Selective hydrogenation of $\alpha, \beta$-unsaturated aldehydes. Catalysis Reviews: Science and Engineering 40, 81-126.

Guo, Z., Chen, Y. T., Li, L. S., Wang, X. M., Haller, G. L., Yang, Y. H., 2010. Carbon nanotubesupported Pt-based bimetallic catalysts prepared by a microwave-assisted polyol reduction method and their catalytic applications in the selective hydrogenation. Journal of Catalysis 276 (2), 314326.

Haaker, M., Verheijen, P., 2004. Local and global sensitivity analysis for a reactor design with parameter uncertainty. Chemical Engineering Research and Design 82 (5), 591-598.

Hadjmohammadi, M., Kamel, K., 2008. Response surface methodology and support vector machine for the optimization of separation in linear gradient elution. Journal of Separation Science 31, 3864-3870.

Huang, K., Chen, F. Q., Lu, D. W., 2001. Artificial neural network-aided design of a multi-component catalyst for methane oxidative coupling. Applied Catalysis A: General 219 (1-2), 61-68.

Ishihara, T., 1987. Hydrogenation of carbon monoxide over $\mathrm{SiO}_{2}$-supported Fe-Co, Co-Ni and Ni-Fe bimetallic catalysts. Applied Catalysis 30 (2), 225-238.

Jones, D. R., 2001. A taxonomy of global optimization methods based on response surfaces. Journal of Global Optimization 21 (4), 345-383.

Kalagnanam, J. R., Diwekar, U. M., 1997. An efficient sampling technique for off-line quality control. Technometrics 39 (3), 308-319.

Kaneeda, M., Iizuka, H., Hiratsuka, T., Shinotsuka, N., Kitahara, Y., Arai, M., 2010. Preparation and screening of various multi-component catalysts for NOx conversion under lean-burn conditions: An 
active and heat-resistant RhPt-NaMn-Ce/ $\mathrm{Al}_{2} \mathrm{O}_{3}$ catalyst. Chemical Engineering Journal 160 (1), 93-98.

Kumar, A., Mukasyan, A. S., Wolf, E. E., 2011. Combustion synthesis of Ni, Fe and Cu multicomponent catalysts for hydrogen production from ethanol reforming. Applied Catalysis A: General $401(1-2), 20-28$.

Lin, J. S., Jang, S. S., 1998. Nonlinear dynamic artificial neural network modeling using an information theory based experimental design approach. Industrial and Engineering Chemistry Research 37, 3640-3651.

Liu, Z. T., Wang, C. X., Liu, Z. W., Lu, J., 2008. Selective hydrogenation of cinnamaldehyde over Pt-supported multi-walled carbon nanotubes: Insights into the tube-size effects. Applied Catalysis A-General 344 (1-2), 114-123.

Lordi, V., Yao, N., Wei, J., 2001. Method for supporting platinum on single-walled carbon nanotubes for a selective hydrogenation catalyst. Chemistry of Materials 13 (3), 733-737.

Lu, G. M., Hoffer, T., Guczi, L., 1992. Reducibility and CO hydrogenation over Pt and Pt-Co bimetallic catalysts encaged in NaY-zeolite. Catalysis Letters 14 (2), 207-220.

McKay, M. D., Beckman, R. J., Conover, W. J., 2000. A comparison of three methods for selecting values of input variables in the analysis of output from a computer code. Technometrics 42 (1), $55-61$.

McRae, G., Tilden, J., Seinfeld, J., 1982. Global sensitivity analysis-a computational implementation of the Fourier amplitude sensitivity test (FAST). Computers and Chemical Engineering 6 (1), 15-25.

Myers, R. H., Montgomery, D. C., 1995. Response Surface Methodology: Process and Product in Optimization Using Designed Experiments. John Wiley and Sons, Inc.

Omata, K., 2011. Screening of New Additives of Active-Carbon-Supported Heteropoly Acid Catalyst for Friedel-Crafts Reaction by Gaussian Process Regression. Industrial \& Engineering Chemistry Research 50 (19), 10948-10954.

Omata, K., Kobayashi, Y., Yamada, M., 2006. Artificial neural network-aided design of Co/SrCO3 catalyst for preferential oxidation of CO in excess hydrogen. Catalysis Today 117, 311-315.

Rasmussen, C. E., 1996. Evaluation of Gaussian processes and other methods for non-linear regression. Ph.D. thesis, University of Toronto, Canada.

Rasmussen, C. E., Williams, C. K. I., 2006. Gaussian Processes for Machine Learning. MIT Press.

Saltelli, A., Ratto, M., Andres, T., Campolongo, F., Cariboni, J., Gatelli, D., Saisana, M., Tarantola, S., 2008. Global Sensitivity Analysis. The Primer. John Wiley and Sons, Inc.

Schanke, D., Vada, S., Blekkan, E. A., Hilmen, A. M., Hoff, A., Holmen, A., 1995. Study of PtPromoted Cobalt CO Hydrogenation Catalysts. Journal of Catalysis 156 (1), 85-95.

Serna, P., Baumes, L. A., Moliner, M., Corma, A., 2008. Combining high-throughput experimentation, advanced data modeling and fundamental knowledge to develop catalysts for the epoxidation of large olefins and fatty esters. Journal of Catalysis 258 (1), 25-34.

Sobol, I. M., 2001. Global sensitivity indices for nonlinear mathematical models and their Monte Carlo estimates. Mathematics and Computers in Simulation 55 (1-3), 271-280, 2nd IMACS Seminar on Monte Carlos Methods, VARNA, BULGARIA, JUN 07-11, 1999.

Tang, Q., Lau, Y., Hu, S., Yan, W., Yang, Y., Chen, T., 2010. Response surface methodology using Gaussian processes: Towards optimizing the trans-stilbene epoxidation over $\mathrm{Co}^{2+}-\mathrm{NaX}$ catalysts. Chemical Engineering Journal 156 (2), 423-431.

Tihay, F., Roger, A. C., Pourroy, G., Kiennemann, A., 2002. Role of the alloy and spinel in the catalytic behavior of $\mathrm{Fe}-\mathrm{Co} /$ cobalt magnetite composites under $\mathrm{CO}$ and $\mathrm{CO}_{2}$ hydrogenation. Energy \& Fuels 16 (5), 1271-1276.

Toebes, M., Nijhuis, T., Hajek, J., Bitter, J., van Dillen, A., Murzin, D., de Jong, K., 2005. Support effects in hydrogenation of cinnamaldehyde over carbon nanofiber-supported platinum catalysts: Kinetic modeling. Chemical Engineering Science 60 (21), 5682-5695.

Tompos, A., Margitfalvi, J. L., Szabo, E. G., Paszti, Z., Sajo, I., Radnoczi, G., 2009. Role of modifiers in multi-component $\mathrm{MgO}$-supported Au catalysts designed for preferential CO oxidation. Journal of Catalysis 266 (2), 207-217. 
Tompos, A., Margitfalvi, J. L., Szabo, E. G., Vegvari, L., 2010. Combinatorial design of $\mathrm{Al}_{2} \mathrm{O}_{3}$ supported Au catalysts for preferential CO oxidation. Topics In Catalysis 53, 108-115.

Valero, S., Argente, E., Botti, V., Serra, J. M., Serna, P., Moliner, M., Corma, A., 2009. DoE framework for catalyst development based on soft computing techniques. Computers and Chemical Engineering 33 (1), 225-238.

Vergunst, T., Kapteijn, F., Moulijn, J., 2001. Kinetics of cinnamaldehyde hydrogenation-concentration dependent selectivity. Catalysis Today 66 (2-4), 381-387.

Yan, W., Chen, Y., Yang, Y., Chen, T., 2011a. Development of high performance catalysts for CO oxidation using data-based modeling. Catalysis Today 174 (1), 127-134.

Yan, W., Hu, S., Yang, Y., Gao, F., Chen, T., 2011b. Bayesian migration of Gaussian process regression for rapid process modeling and optimization. Chemical Engineering Journal 166 (3), 1095-1103.

Yuan, J., Wang, K. S., Yu, T., Fang, M. L., 2008. Reliable multi-objective optimization of high-speed WEDM process based on Gaussian process regression. International Journal of Machine Tools and Manufacture 48 (1), 47-60. 


\section{List of Tables}

$1 \quad$ Initial experiments. . . . . . . . . . . . . . . . . . . . . . . . . 19

2 Results for iterative modeling and optimization. . . . . . . . . . 20

3 SI of the main effects. . . . . . . . . . . . . . . . . . 21

4 SI of the second order interactions. . . . . . . . . . . . . . 22

5 Particle size calculated from XRD patterns. . . . . . . . . . . 23

\section{List of Figures}

1 Reaction pathways for hydrogenation of CALD. . . . . . . . . . . . . . 24

2 The main effect, $E\left(y \mid x_{i}\right)$, against component loadings, for conversion, selectivity and yield. . . . . . . . . . . . . . . . 25

3 The expected response surface of conversion, $E\left(y \mid x_{i}, x_{j}\right)$, against pairs of component loadings. . . . . . . . . . . . . . . . . 26

4 The expected response surface of selectivity, $E\left(y \mid x_{i}, x_{j}\right)$, against pairs of component loadings. . . . . . . . . . . . . . . . . . . 27

5 The expected response surface of yield, $E\left(y \mid x_{i}, x_{j}\right)$, against pairs of component loadings. . . . . . . . . . . . . . . . . . . . . . 28

6 XRD patterns of catalysts No. 11-13 and 15. . . . . . . . . . . 29

$7 \quad$ TEM images and particle size distributions of catalysts (a) No. 11, (b) No. 12, (c) No. 13 and (d) No. 15. . . . . . . . . . . . . . . 30

8 XPS spectra of (a) Pt, (b) Co and (c) Fe on catalysts No. 11-13 and 15. 31 
Table 1: Initial experiments.

\begin{tabular}{ccccccc}
\hline No. & $x_{1}(\mathrm{Pt})$ & $x_{2}(\mathrm{Co})$ & $x_{3}(\mathrm{Fe})$ & Conversion & Selectivity & Yield \\
\hline 1 & 4.80 & 2.00 & 2.33 & 0.771 & 0.741 & 0.571 \\
2 & 4.60 & 2.50 & 1.67 & 0.744 & 0.716 & 0.533 \\
3 & 4.40 & 1.50 & 2.78 & 0.729 & 0.747 & 0.545 \\
4 & 4.20 & 2.75 & 2.11 & 0.852 & 0.727 & 0.619 \\
5 & 4.00 & 1.75 & 1.44 & 0.623 & 0.714 & 0.444 \\
6 & 3.80 & 2.25 & 2.56 & 0.457 & 0.861 & 0.394 \\
7 & 3.60 & 1.25 & 1.89 & 0.642 & 0.767 & 0.493 \\
8 & 3.40 & 2.88 & 1.22 & 0.862 & 0.750 & 0.647 \\
9 & 3.20 & 1.88 & 2.93 & 0.764 & 0.806 & 0.615 \\
10 & 3.00 & 2.38 & 2.26 & 0.786 & 0.783 & 0.615 \\
\hline
\end{tabular}


Table 2: Results for iterative modeling and optimization.

\begin{tabular}{ccccccccc}
\hline No. & $x_{1}(\mathrm{Pt})$ & $x_{2}(\mathrm{Co})$ & $x_{3}(\mathrm{Fe})$ & $\hat{y}$ & $\mathrm{EI}_{\max }$ & Conversion & Selectivity & Yield \\
\hline 11 & 5.00 & 3.00 & 1.00 & $0.569 \pm 0.653$ & 0.223 & 0.775 & 0.712 & 0.552 \\
12 & 5.00 & 1.00 & 3.00 & $0.564 \pm 0.593$ & 0.196 & 0.782 & 0.750 & 0.587 \\
13 & 3.00 & 1.00 & 1.00 & $0.593 \pm 0.455$ & 0.156 & 0.786 & 0.780 & 0.613 \\
14 & 3.40 & 1.00 & 3.00 & $0.638 \pm 0.226$ & 0.086 & 0.860 & 0.828 & 0.712 \\
15 & 3.40 & 1.30 & 2.60 & $0.744 \pm 0.057$ & 0.034 & 0.861 & 0.864 & 0.744 \\
$16^{*}$ & 4.20 & 1.00 & 1.00 & $0.593 \pm 0.283$ & 0.011 & - & - & - \\
\hline
\end{tabular}

* This designed experiment was not conducted. 
Table 3: SI of the main effects.

\begin{tabular}{|c|c|c|c|c|c|c|}
\hline \multirow[t]{2}{*}{ Factor } & \multicolumn{2}{|c|}{ Conversion } & \multicolumn{2}{|c|}{ Selectivity } & \multicolumn{2}{|c|}{ Yield } \\
\hline & $S_{i}$ & $S_{T_{i}}$ & $S_{i}$ & $S_{T_{i}}$ & $\overline{S_{i}}$ & $S_{T_{i}}$ \\
\hline Pt loac & 0.763 & 0.831 & 0.069 & 0.204 & 0.473 & 0.604 \\
\hline Co loa & 0.023 & 0.088 & 0.022 & 0.065 & 0.002 & 0.097 \\
\hline Fe loading & 0.131 & 0.155 & 0.735 & 0.887 & 0.381 & 0.455 \\
\hline
\end{tabular}


Table 4: SI of the second order interactions.

\begin{tabular}{ccccc}
\hline \multirow{5}{*}{ Conversion } & & $\mathrm{Pt}$ & $\mathrm{Co}$ & $\mathrm{Fe}$ \\
& $\mathrm{Pt}$ & - & 0.062 & 0.007 \\
& $\mathrm{Co}$ & 0.062 & - & 0.003 \\
& $\mathrm{Fe}$ & 0.007 & 0.003 & - \\
\hline \multirow{5}{*}{ Selectivity } & $\mathrm{Pt}$ & - & 0.010 & 0.131 \\
& $\mathrm{Co}$ & 0.010 & - & 0.013 \\
& $\mathrm{Fe}$ & 0.131 & 0.013 & - \\
\hline \multirow{5}{*}{ Yield } & & $\mathrm{Pt}$ & $\mathrm{Co}$ & $\mathrm{Fe}$ \\
& $\mathrm{Pt}$ & - & 0.063 & 0.054 \\
& $\mathrm{Co}$ & 0.063 & - & 0.004 \\
& $\mathrm{Fe}$ & 0.054 & 0.004 & - \\
\hline
\end{tabular}


Table 5: Particle size calculated from XRD patterns.

\begin{tabular}{ccccc}
\hline No. & $x_{1}(\mathrm{Pt})$ & $x_{2}(\mathrm{Co})$ & $x_{3}(\mathrm{Fe})$ & Particle size $(\mathrm{nm})$ \\
\hline 11 & 5.00 & 3.00 & 1.00 & 7.0 \\
12 & 5.00 & 1.00 & 3.00 & 5.8 \\
13 & 3.00 & 1.00 & 1.00 & 6.1 \\
15 & 3.40 & 1.30 & 2.60 & 5.1 \\
\hline
\end{tabular}




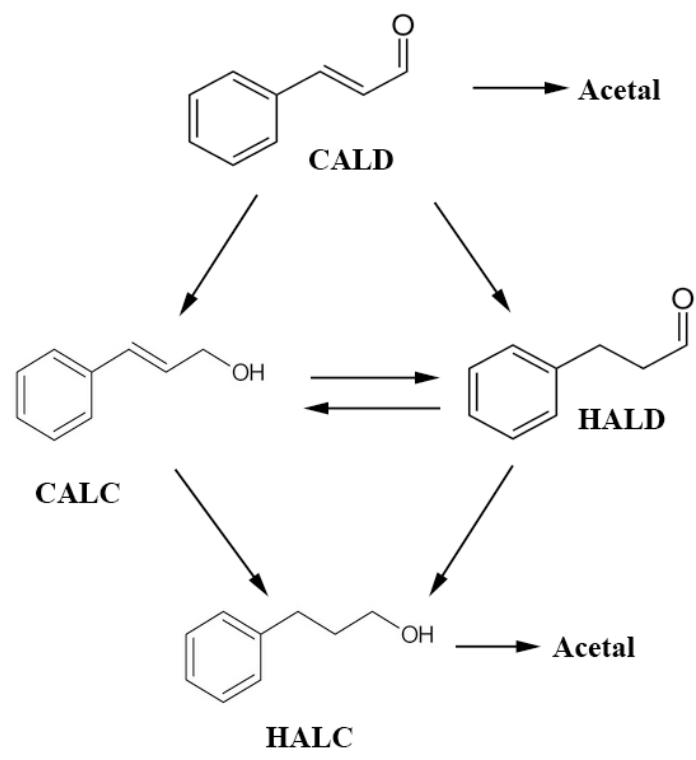

+Dehydronation products

Condensation products

Figure 1: Reaction pathways for hydrogenation of CALD. 

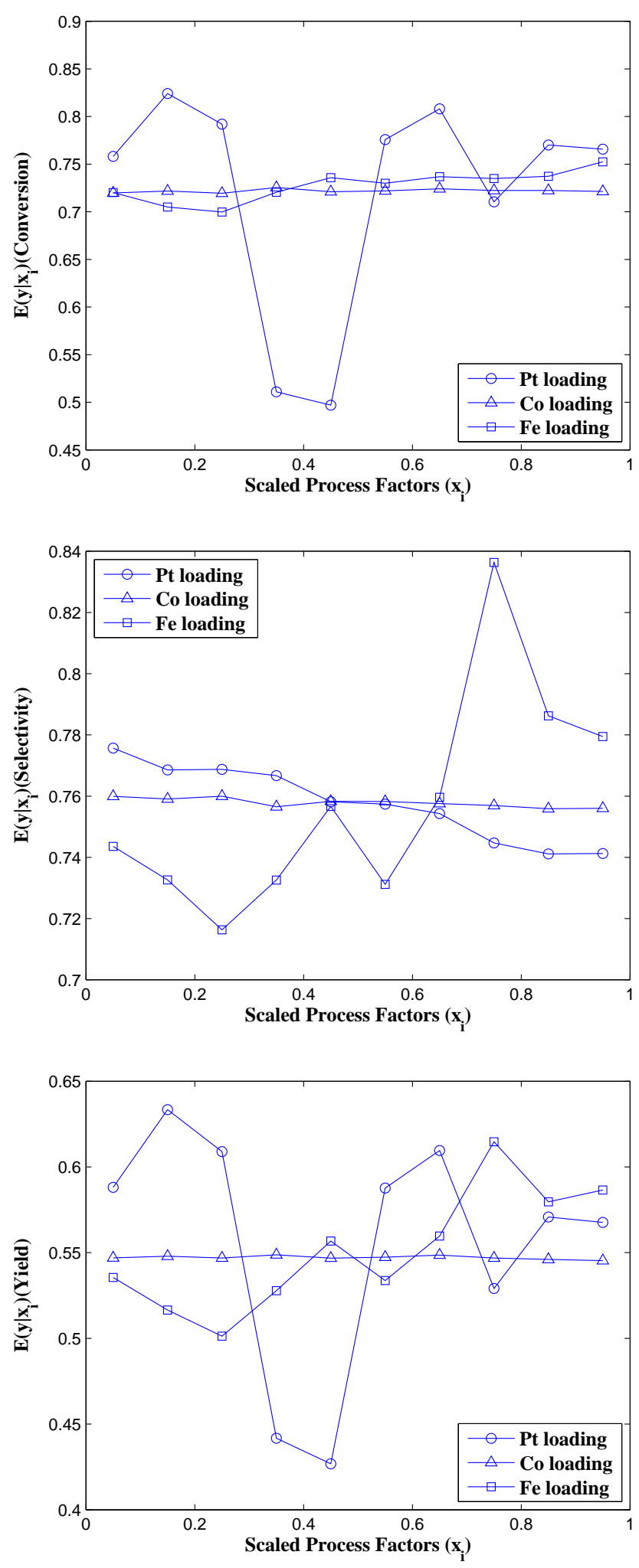

Figure 2: The main effect, $E\left(y \mid x_{i}\right)$, against component loadings, for conversion, selectivity and yield. 

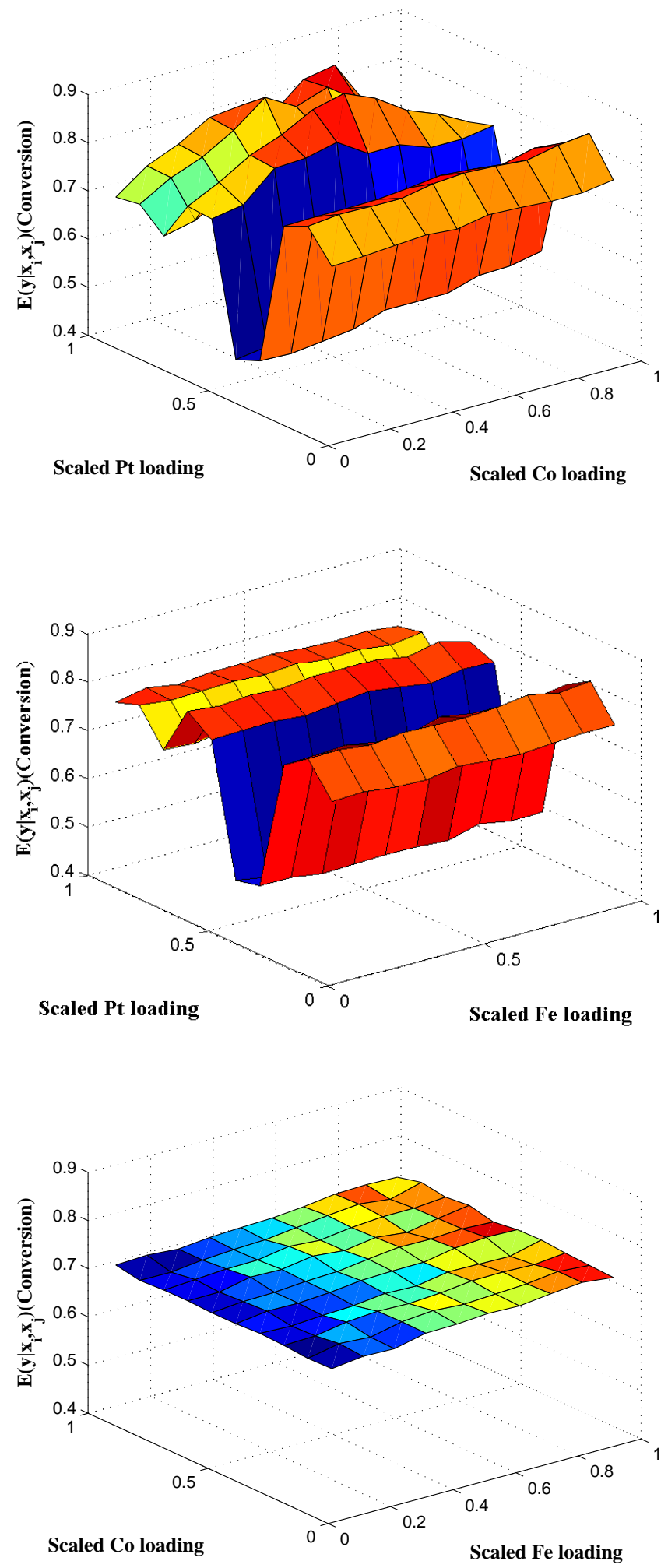

Figure 3: The expected response surface of conversion, $E\left(y \mid x_{i}, x_{j}\right)$, against pairs of component loadings. 



Figure 4: The expected response surface of selectivity, $E\left(y \mid x_{i}, x_{j}\right)$, against pairs of component loadings. 

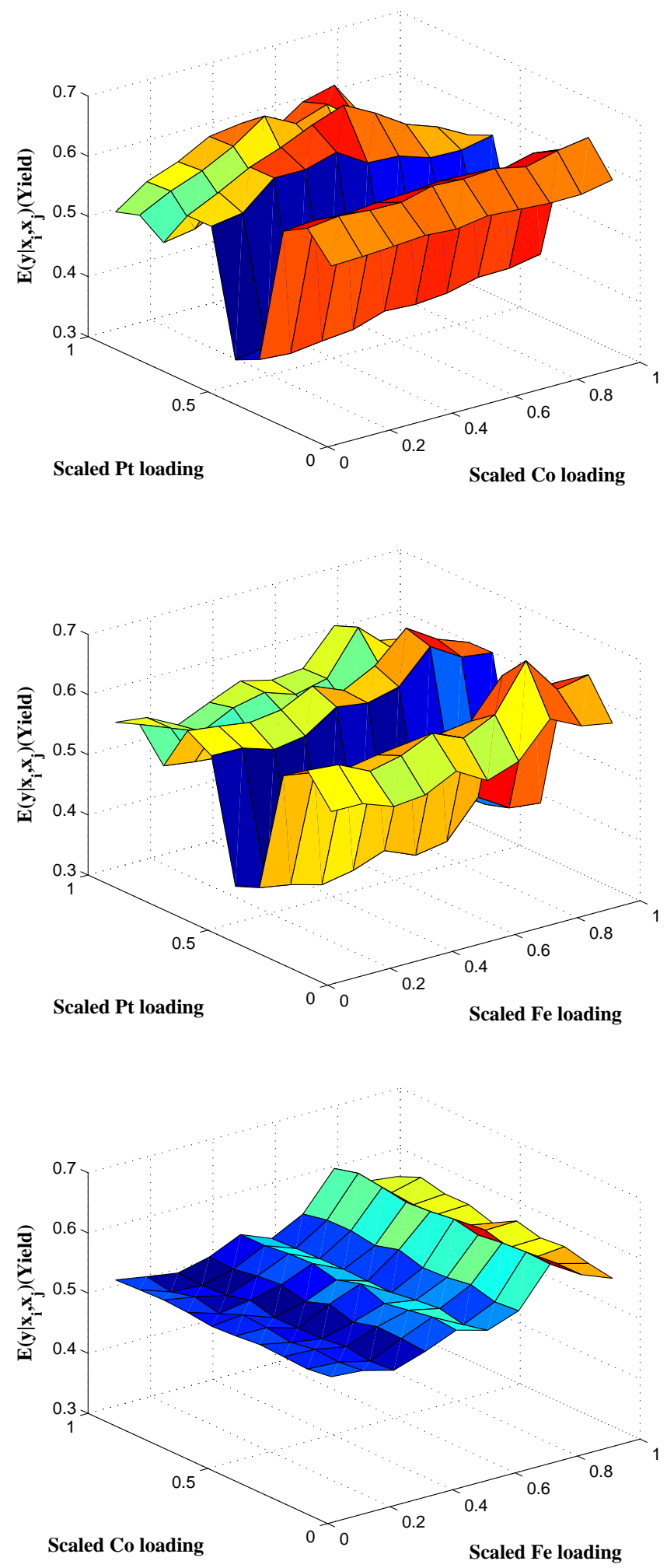

Figure 5: The expected response surface of yield, ${ }_{28} E\left(y \mid x_{i}, x_{j}\right)$, against pairs of component loadings. 


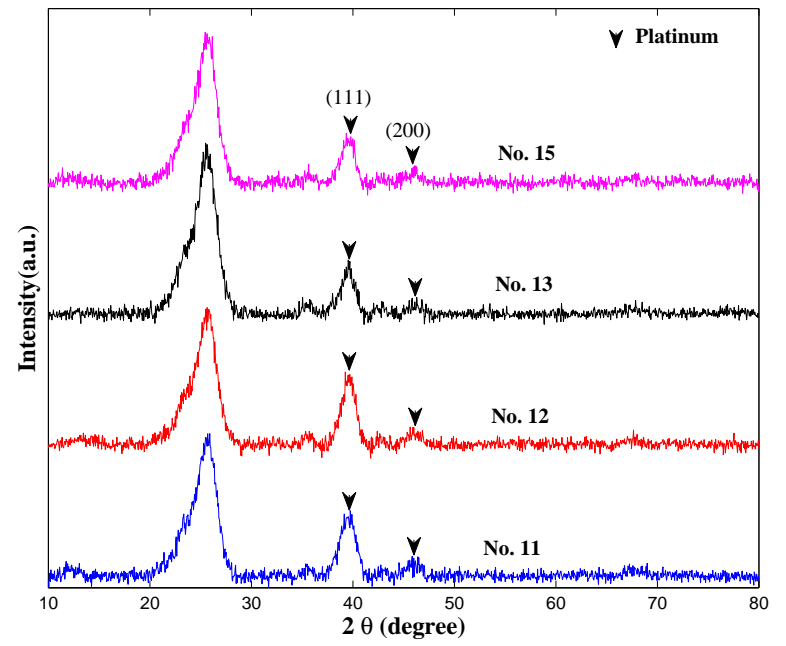

Figure 6: XRD patterns of catalysts No. 11-13 and 15. 

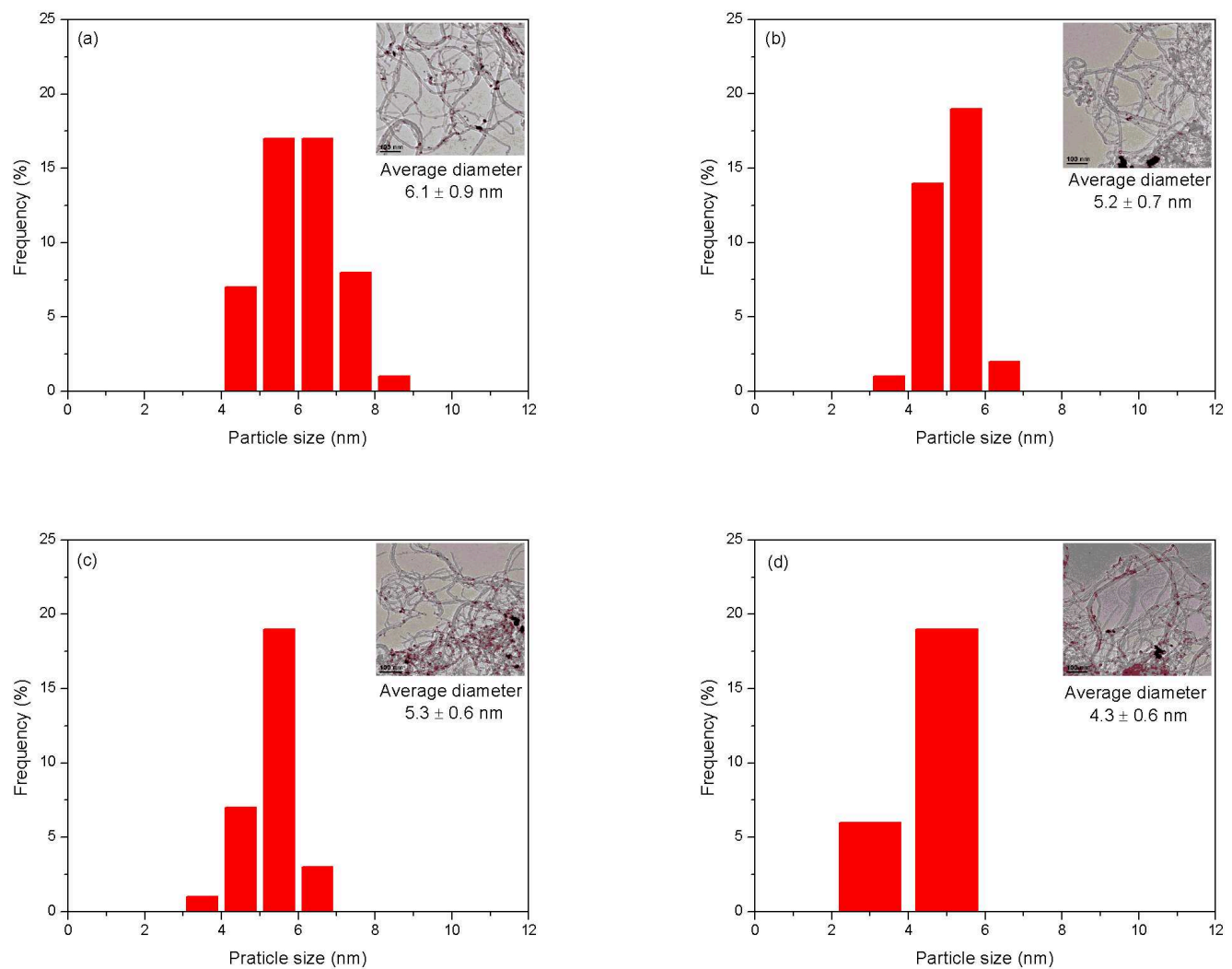

Figure 7: TEM images and particle size distributions of catalysts (a) No. 11, (b) No. 12, (c) No. 13 and (d) No. 15. 

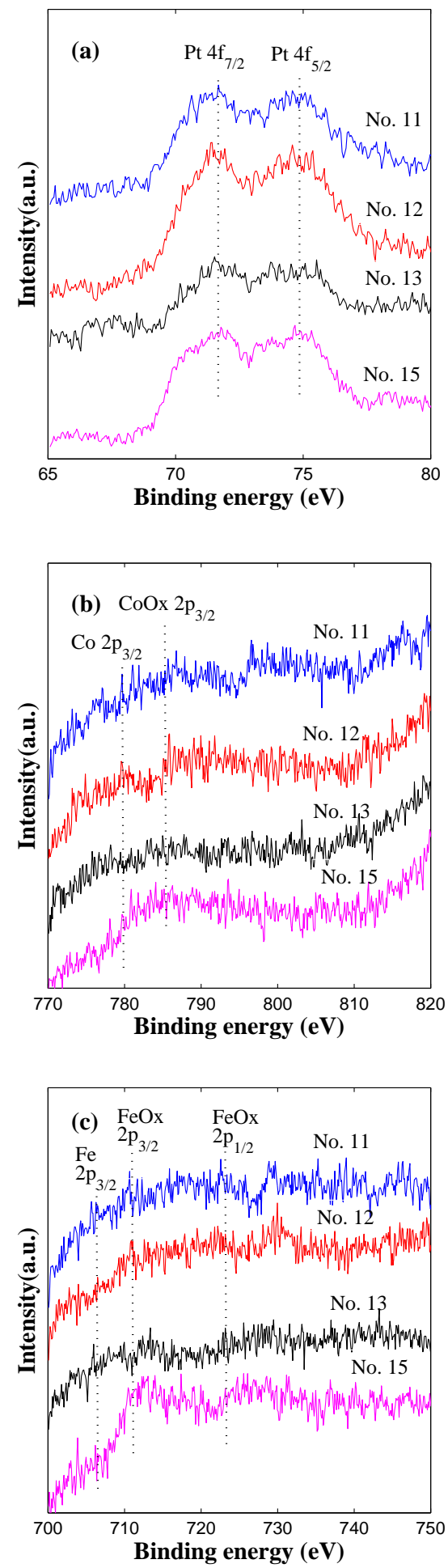

Figure 8: XPS spectra of (a) Pt, (b) Co and (c) Fe on catalysts No. 11-13 and 15. 